\title{
Otx2 Controls Identity and Fate of Glutamatergic Progenitors of the Thalamus by Repressing GABAergic Differentiation
}

\author{
Eduardo Puelles, ${ }^{1}$ Dario Acampora, ${ }^{1,2,4}$ Robindra Gogoi, ${ }^{1}$ Francesca Tuorto, ${ }^{2,4}$ Alessandro Papalia, ${ }^{1}$ \\ Francois Guillemot, ${ }^{5}$ Siew-Lan Ang, ${ }^{5}$ and Antonio Simeone $e^{1,2,3,4}$ \\ ${ }^{1}$ Medical Research Council Centre for Developmental Neurobiology, King's College London, London SE1 1UL, United Kingdom, ${ }^{2}$ CEINGE Biotecnologie \\ Avanzate, 80145 Naples, Italy, ${ }^{3}$ Scuola Europea di Medicina Molecolare, 80145 Naples, Italy, ${ }^{4}$ Institute of Genetics and Biophysics "A. Buzzati-Traverso," \\ Consiglio Nazionale delle Ricerche, 80131 Naples, Italy, and "Division of Developmental Neurobiology, National Institute for Medical Research, London \\ NW7 1AA, United Kingdom
}

\begin{abstract}
GABAergic and glutamatergic neurons modulate inhibitory and excitatory networks in the CNS, and their impairment may cause neurological and psychiatric disorders. Thus, understanding the molecular mechanisms that control neurotransmitter phenotype and identity of excitatory and inhibitory progenitors has considerable relevance. Here we investigated the consequence of Otx2 (orthodenticle homolog) ablation in glutamatergic progenitors of the dorsal thalamus (referred to as thalamus). We report that 0tx2 is cellautonomously required in these progenitors to repress GABAergic differentiation. Our data indicate that Otx2 may prevent GABAergic fate switch by repressing the basic helix-loop- helix gene Mash1 (mammalian achaete-schute homolog) in progenitors expressing Ngn2 (neurogenin homolog). The lack of Otx2 also resulted in the activation of Pax3 (paired box gene), Pax7, and Lim1 (Lin-11/Isl-1/Mec-3), three genes normally coexpressed with Mash1 and GABAergic markers in the pretectum, thus suggesting that thalamic progenitors lacking 0tx2 exhibit marker similarities with those of the pretectum. Furthermore, 0tx2 ablation gave rise to a marked increase in proliferating activity of thalamic progenitors and the formation of hyperplastic cell masses. Thus, this study provides evidence for a novel and crucial role of 0tx2 in the molecular mechanism by which identity and fate of glutamatergic precursors are established in the thalamus. Our data also support the concept that proper assignment of identity and fate of neuronal precursors occurs through the suppression of alternative differentiation programs.
\end{abstract}

Key words: Otx2; neuronal differentiation; glutamatergic fate; GABAergic fate; proliferation; thalamus

\section{Introduction}

Regionalization and assignment of neuronal fates are complex events determined by a genetic cascade of interactions conferring a progressive restriction of cell type (Wolpert, 1969; Lumsden and Krumlauf, 1996; Rubenstein et al., 1998; Briscoe et al., 2000; Jessell, 2000; Briscoe and Ericson, 2001). In this process, regionally restricted identities are thought to be maintained through the formation of compartments whose positional identity is ensured by a polyclonal cell population with restricted cell lineages

Received March 14, 2006; revised April 19, 2006; accepted April 19, 2006.

This work was supported by Medical Research Council (MRC) Grant G9900955, Wellcome Trust Grant 062642/ Z100, Italian Association for Cancer Research, Eubiotech Grant QLG3-CT-2000-023109, Fondo per gli Investimenti della Ricerca di Base (FIRB) Genotopo, FIRB Neuroscienze, Ministero dell'Istruzione, dell'Università, e della Ricerca (DM 8/10/96, number 623), and the Fondation Bettencourt Schueller (all to A.S.), by MRC Grant G117/978 (to D.A.), and by grants from the European Community Biotech Programme, Institut National de la Santé et de la Recherche Médicale, Centre National de la Recherche Scientifique, Hôpital Universitaire de Strasbourg, and MRC (all to S.-L.A.). We thank A. Lumsden, L. Puelles, T. Russo, and R. Di Lauro for critical reading of this manuscript and helpful discussions. We are also indebted to D. Anderson and G. Corte for the generous gift of the $\mathrm{Ngn} 2$ and $0 \mathrm{tx} 2$ antibodies, respectively.

Correspondence should be addressed to Antonio Simeone, CEINGE Biotecnologie Aranzate, Via Comunale Margherita 482, 80145 Naples, Italy. E-mail: antonio.simeone@kcl.ac.uk.

E. Puelles's present address: Instituto de Neurociencias de Alicante, Universidad Miguel Hernández, Consejo Superior de Investigaciones Científicas, Campus de San Joan, Apartado de correos, 18, 03550 San Joan, Alicante, Spain.

DOI:10.1523/JNEUROSCI.1097-06.2006

Copyright $\odot 2006$ Society for Neuroscience $\quad$ 0270-6474/06/265955-10\$15.00/0
(Lumsden, 1990; Figdor and Stern, 1993). These events are controlled by inducing signals and depend on the responding ability of target cells (Ye et al., 1998; Edlund and Jessell, 1999; Hynes and Rosenthal, 1999; Jessell, 2000; Agarwala et al., 2001; Briscoe and Ericson, 2001; Wurst and Bally-Cuif, 2001; Kiecker and Lumsden, 2004). Patterning signals are converted into positional identity by specific transcription factors whose regionally restricted expression provides neuronal precursors with a precise molecular identity. On this basis, neurons with distinct neurotransmitter phenotypes originate from progenitors located in restricted areas and characterized by a typical molecular code of selector genes (Edlund and Jessell, 1999; Jessell, 2000; Briscoe and Ericson, 2001).

Excitatory and inhibitory neurons, which represent the two major neuronal cell types, are functionally defined by the mutually exclusive expression of glutamate and GABA neurotransmitters, are generated in different areas along the CNS, and are involved in the pathophysiology of several neurological and psychiatric disorders (Bennett and Balcar, 1999). In particular, the GABAergic and glutamatergic neurons of the forebrain arise from different pools of progenitors. GABAergic neurons are generated principally in the basal telencephalon, prethalamus, and pretectum, whereas glutamatergic neurons arise from the dorsal telencephalon and dorsal thalamus (referred to as thalamus) 
(Luskin et al., 1993; Mione et al., 1994; Puelles and Rubenstein, 2003). However, the genetic program that controls the generation of GABAergic and glutamatergic neurons, the choice between the inhibitory and excitatory fate and the molecular basis of their mutually exclusive activation, is far from being fully understood.

Nevertheless, the functional analysis of several transcription factors expressed selectively in GABAergic or glutamatergic progenitors in the forebrain has suggested the existence of a genetic program suppressing GABAergic differentiation in glutamatergic progenitors (Casarosa et al., 1999; Fode et al., 2000; Toresson et al., 2000; Muzio et al., 2002; Parras et al., 2002; Miyoshi et al., 2004; Schuurmans et al., 2004).

Moreover in the spinal cord, it has been shown recently that the transcription factors $T l x 1$ and $T l \times 3$ operate as postmitotic selectors required to promote glutamatergic fate and repress GABAergic differentiation (Cheng et al., 2004).

Otx1 and Otx2 (orthodenticle homologs), two homeodomain transcription factors, are required for regionalization and patterning of forebrain and midbrain (Acampora and Simeone, 1999; Simeone et al., 2002; Puelles et al., 2003, 2004; Simeone, 2005). In this study, we investigated through conditional mutagenesis the functional consequence of Otx2 inactivation in progenitors of the thalamus that are programmed normally to generate glutamatergic neurons. We show that Otx2 ablation in these progenitors results in the loss of glutamatergic differentiation, switch to GABAergic fate, and increased proliferating activity.

Thus, these findings indicate that, in the thalamus, Otx2 is required to control identity and fate of glutamatergic precursors by suppressing the GABAergic differentiation.

\section{Materials and Methods}

Generation of mouse mutants and genotyping. The generation of $\mathrm{Otx} 1^{\text {cre/+ }}$ and Otx $2^{f l o x /+}$ mouse strains as well as their genotyping procedure have been reported previously in detail (Puelles et al., 2003).

In situ hybridization and immunohistochemistry. In situ hybridization was performed as described previously (Simeone, 1999). Probes for Otx2s, Cre (Enterobacteria phage P1, cyclization recombinase), Gli1 (glioma-associated oncogene homolog), Gli2, Gli3, Smo (smoothened homolog), Ptch1 (patched homolog), Lim1 (Lin-11/Isl-1/Mec-3), Pax3 (paired box gene), Pax7, Dlx1 (distal-less homeobox), Dlx5, Emx1 (empty spiracles homolog), Tbr1 (T-box brain gene), Nkx2.1 (NK2 transcription factor-related), $N k \times 2.2, N k \times 6.1, G b \times 2$ (gastrulation brain homeobox), Shh (sonic hedgehog), Fgf8 (fibroblast growth factor), Wnt1 (wingless-type MMTV integration site family), Wnt3a, Bmp2 (bone morphogenic protein), Bmp6, Isl1 (islet), Foxa2 (forkhead box), Msx1 (msh homeobox homolog), VGLUT2 (vesicular glutamate transporter), Gad1 (glutamic acid decarboxylase), TH (tyrosine hydroxylase), $D B H$ (dopamine $\beta$-hydroxylase), and 5-HT (5-hydroxytryptamine) have been described previously (Puelles et al., 2003, 2004) or correspond to PCR fragments ranging between 0.5 and $1 \mathrm{~kb}$. For immunohistochemistry, only sections from wax-embedded embryos were used. Antigens were unmasked by four rounds of microwave boiling ( $4 \mathrm{~min} /$ boiling) at $700 \mathrm{~W}$ in sodium citrate buffer (10 mM), pH 6 (Shi et al., 1991, 1997, 2001), and one or two primary antibodies, diluted at the appropriate concentration in blocking solution, were applied to the sections and left overnight at room temperature. After washing in PBS, secondary antibodies were applied for $60 \mathrm{~min}$, and then the slides were PBS washed and processed for carbazole or DAB staining or, in the case of fluorescent secondary antibodies, for their observation and image capture.

For multiple rounds of fluorescent immunohistochemistry, we set up a modification of the common procedure described above that allowed us to detect four or more antibodies in the cells of the same section. This modification aims at detecting more genes in the same cell to define unambiguously a complex molecular code, if any. Based on our modification, after the first round of fluorescent immunohistochemistry per- formed as described above, the sections of interest were selected and their images captured; then the slides were boiled again four times in sodium citrate buffer to remove primary and secondary antibodies, checked for lack of fluorescence, and probed with a second pair of primary antibodies. Then appropriate secondary antibodies were applied, and the images of the same sections selected in the first round were captured for the second pair of antibodies. For each of the following rounds, if required, the sections were boiled again four times, checked for the lack of fluorescence, and processed as above described. Although this procedure has been used up to five sequential rounds for a maximum of 10 different primary antibodies, in this study, sections were used for a maximum of two sequential rounds. In this modified procedure, lack of fluorescence indicated that secondary antibodies, but not necessarily also the primary antibodies, had been removed. To rule out this possibility, control slides immunostained with the same primary and secondary antibodies were boiled and hybridized in parallel only with secondary antibodies to check the absence of staining and, thereby, the absence of residual primary antibodies. We also tested that antibodies used in the first round efficiently worked after the last round of immunohistochemistry, thus proving that, during the procedure, antigens were not severely affected. Importantly, we preselected the primary antibodies for their efficiency and used those with the highest antigen affinity, and thereby more difficult to be removed, in the last round of immunohistochemistry [e.g., $\alpha$-bromodeoxyuridine ( $\alpha \mathrm{BrdU})$ ].

Antibodies. The rabbit antibodies were directed against Otx2 (1:5000; G. Corte, National Institute for Cancer Research, Genova, Italy), Pax3 (1:200; Zymed, San Francisco, CA), GABA (1:500; Sigma, St. Louis, MO), Lim1 (1:200; Chemicon, Temecula, CA), VGLUT2 (1:300; SySy, Goettingen, Germany), and $\mathrm{Ph}-\mathrm{H} 3$ (the phosphorylated form of histone $\mathrm{H} 3$ ) (1:200; Upstate, Charlottesville, VA). The mouse antibodies were directed against GFAP (1:200; Chemicon), 5-HT (1:100; Chemicon), TH (1:300; Chemicon), neurofilament (1:100; Developmental Studies Hybridoma Bank, University of Iowa, Iowa City, IA), Lim1 (1:100; Developmental Studies Hybridoma Bank), Mash1 (mammalian achaeteschute homolog) (1:10; F. Guillemot, National Institute for Medical Research, London, UK), Ngn2 (neurogenin) (1:25; D. Anderson, Howard Hughes Medical Institute, Pasadena, CA), Pax7 (1:100; Developmental Studies Hybridoma Bank), and BrdU (1:100) (Becton Dickinson, Mountain View, CA). The goat antibody was directed against Pax3/7 (1:100; Santa Cruz Biotechnology), the sheep antibody was directed against BrdU (1:200; Abcam, Cambridge, MA), and the guinea pig antibody was directed against VGLUT2 (1:500; Chemicon).

Analysis of the cell patches: size, location, and expression pattern. For the anatomo-histological analysis aimed at studying size and location of the patches, we analyzed six conditional mutants at embryonic day 12.5 (E12.5), five at E13.5, and six at E16.5. The same number of wild-type and Otx $1^{\text {crel+}}$; Otx $2^{+/-}$control embryos was analyzed at the same stages. Embryos were wax embedded, sectioned, and Nissl stained. Every fourth serial section for E12.5 and E13.5 embryos and every sixth serial section for E16.5 embryos were selected and analyzed for the size and location of cell patches. Three different sizes were arbitrarily defined: the small size corresponded to the cell patches that were one-half or less of those with intermediate size, which were, in turn, between one-half and one-fourth of those with the large size.

For in situ hybridization analysis with radiolabeled probes, a total of 15 conditional mutant embryos at E12.5 and 11 at E16.5 were analyzed together with a corresponding number of $\mathrm{Otx} 1^{\mathrm{cre} /+} ; \mathrm{Otx} 2^{+/-}$control embryos.

For the analysis of cell patches in terms of expression pattern and relative distribution of $\mathrm{Lim} 1^{+}$and $\mathrm{Pax} 3 / 7^{+}$-Otx $2^{-}$cells, six conditional mutants were studied at E12.5 and E16.5, respectively. After the immunostaining with Otx2, Pax3/7, and Lim1, 115 patches at E12.5 and 105 at E16.5 were scored to inspect whether the same general pattern of expression reported in Figure 4 was detected in all of the patches. For numerical analysis, 80 patches immunostained for Lim 1 and Pax3/7 were selected and analyzed at E12.5 and E16.5, respectively. Images of these patches were captured, cells were counted, and the ratio between $\operatorname{Lim} 1^{+}$and Pax $3 / 7^{+}-$Otx $2^{-}$cells was determined.

Cell proliferation experiments and cell counting. For short-pulse exper- 

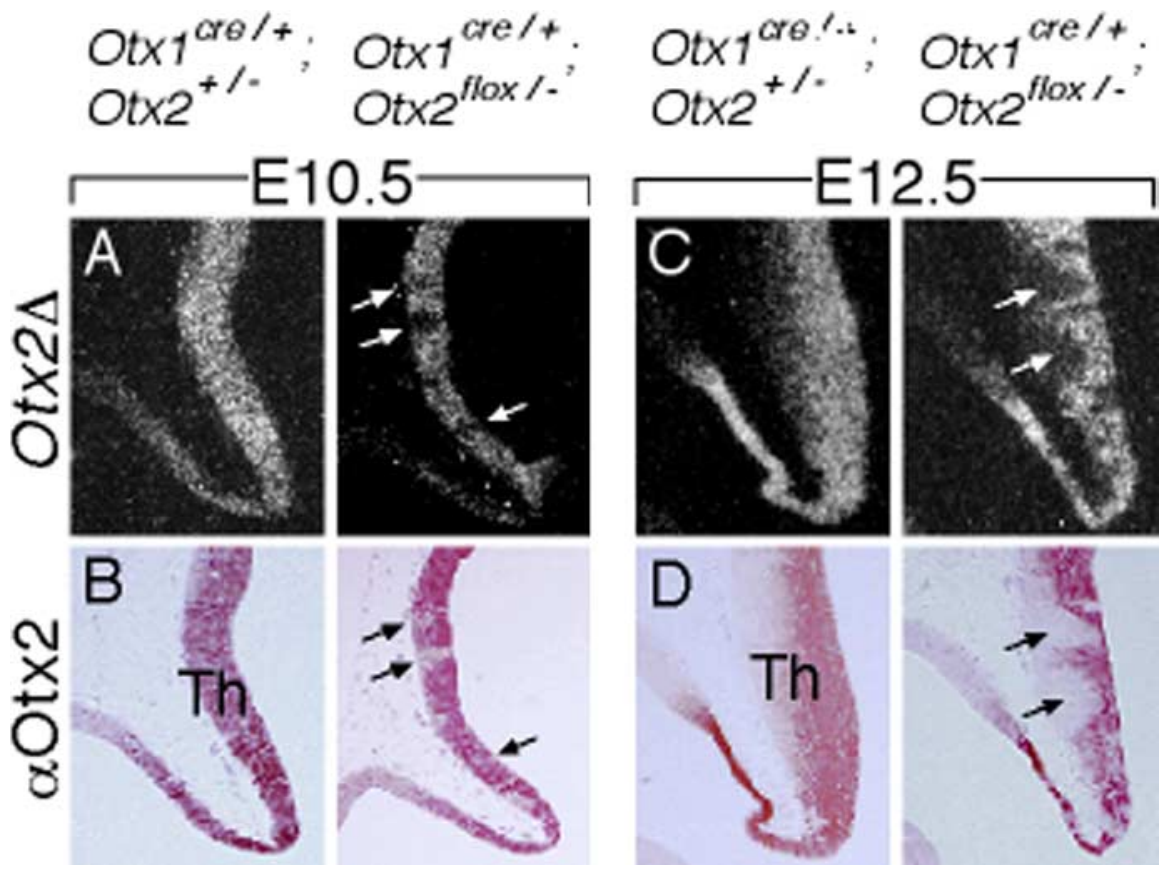

Figure 1. Inactivation of the $0 t \times 2^{f l o x}$ allele by the $0 t x 1$-driven Cre recombinase. $A-D$, In situ hybridization with the $0 t \times 2 \Delta$

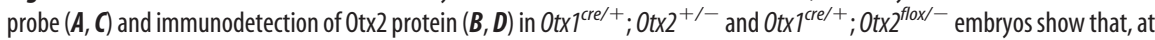
E10.5, the $0 t \times 22^{\text {flox }}$ allele is inactivated in small groups of progenitors (arrows in $\boldsymbol{A}, \boldsymbol{B}$ ), which, at E12.5, may increase in size (arrows in $(, D)$. Th, Thalamus.

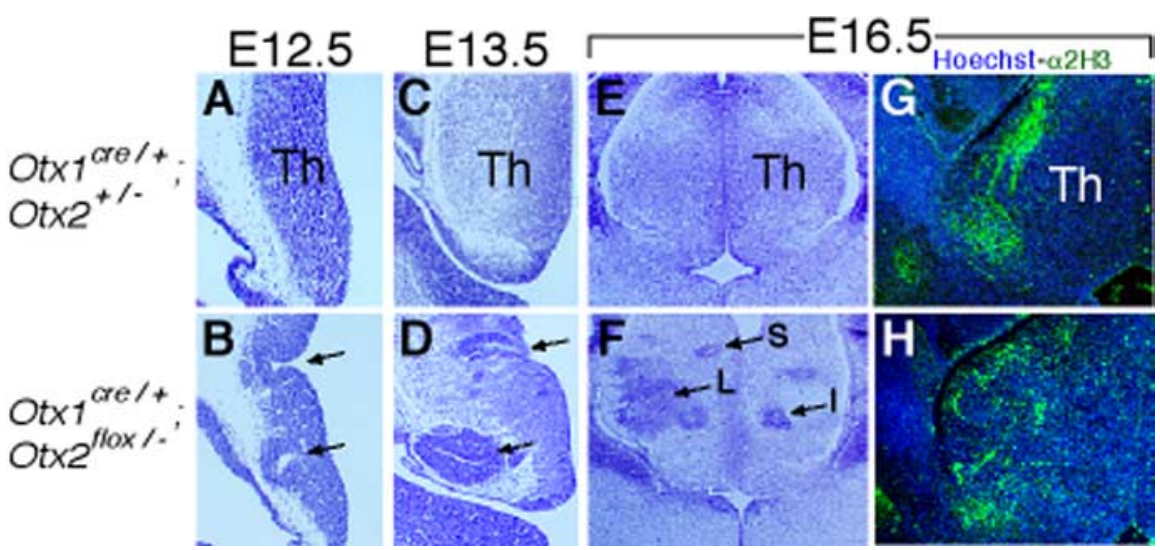

Figure 2. Anatomo-histological abnormalities in the thalamus of conditional mutants. $A-F$, Nissl staining of frontal sections through the thalamus of $O t x 7^{\text {cre/ }+} ; \operatorname{Otx}^{+/-}(\boldsymbol{A}, \boldsymbol{C}, \boldsymbol{E})$ and conditional mutants $(\boldsymbol{B}, \boldsymbol{D}, \boldsymbol{F})$ at E12.5, E13.5, and E16.5. The arrows in $\boldsymbol{B}, \boldsymbol{D}$, and $\boldsymbol{F}$ point to swellings and/or cell masses within the thalamus. $\mathbf{G}, \boldsymbol{H}$, Neurofilament immunodetection $(\alpha 2 \mathrm{H} 3)$ in frontal sections counterstained with Hoechst reveals that, compared with control embryos $(\boldsymbol{G})$, conditional mutants $(\boldsymbol{H})$ exhibit heavy abnormalities of axonal routes in the thalamus. Th, Thalamus; S, L, and I, cell masses of small, large, and intermediate size, respectively.

analyzed. After a BrdU pulse of $60 \mathrm{~min}$, the ratio between the mean of the $\mathrm{BrdU}^{+}$cell number detected in the patches and that of control embryos gives a numerical estimation of the DNA synthesizing activity ( $\mathrm{S}$ phase). The same procedure was used for $\mathrm{Ph}-\mathrm{H}^{+}{ }^{+}$cells to compare the mitotic activity (M phase) of the patches with that of control embryos.

\section{Results}

Otx2 inactivation in the thalamus of Otx $1^{\text {cre/+ }} ;$ Otx $2^{\text {flox/- }}$ mutants

To study the role of $O t x 2$ in the differentiation of thalamic progenitors, Otx2 was inactivated by Otx1-driven Cre recombinase activity. The generation of the $O t x 1^{\text {cre }}$ and $O t x 2^{f l o x}$ mouse models has been reported previously (Puelles et al., 2003).

To define the areas of coexpression, we compared the distribution of Otxl, Cre, and Otx2 transcripts in the thalamus of wild-type and Otx $1^{\text {cre/+ }}$ mutants. No difference in expression pattern was detected between Otxl and Cre in Otx $1^{\text {cre/+ }}$ embryos (data not shown). In these embryos, Otx1, Cre, and Otx2 were coexpressed in the thalamic neuroepithelium at E9.5, E10.5, and E12.5 (data not shown) (Simeone et al., 1992, 1993; Puelles et al., 2003). In particular, at E12.5, their coexpression in the thalamus was primarily restricted to the ventricular and subventricular zones (Simeone et al., 1993) (data not shown). Otx2 inactivation in this territory was monitored by analyzing the distribution of the Otx2 protein and that of the Otx2 unfloxed transcripts using the Otx2 antibody and the Otx $2 \Delta$ probe, respectively. The Ot $x 2 \Delta$ probe detected only Otx 2 unfloxed transcripts and has been described previously (Puelles et al., 2003, 2004). This analysis showed that, in Otx $1^{\text {cre/+ }} ;$ Otx $2^{\text {flox/- }}$ mutants, Otx2 was first inactivated at E10.5 in restricted groups of neuronal precursors (Fig. 1A,B, arrows), which, at E12.5, increased in number and showed heterogeneity in size (Fig. $1 C, D$, arrows and data not shown). Therefore, as reported previously for the midbrain (Puelles et al., 2003), Otx1-driven Cre recombinase showed a limited efficiency also

iments, pregnant females at E10.5, E12.5, E13.5, or E16.5 were killed 60 min after intraperitoneal injection with BrdU solution $(50 \mathrm{mg} / \mathrm{kg}$ body weight). For long-pulse experiments, BrdU solution was administered in four sequential (every $2 \mathrm{~h}$ from E13.3 to E13.6) intraperitoneal injections to pregnant females that were killed at E16.5. To determine the numerical increase of $\mathrm{BrdU}^{+}$and $\mathrm{Ph}-\mathrm{H}_{3}{ }^{+}$cells in conditional mutants at E13.5, sections of patches containing the ventricle-like cavity similar to that shown in Figure 5, $K, O$, and $S$, were selected from five embryos. The area selected for $\mathrm{BrdU}^{+}$and $\mathrm{Ph}-\mathrm{H}_{3}{ }^{+}$cell counting was a rectangular region having as the major side the neuroepithelial border facing the ventriclelike cavity of the patches. A total of 10 patches of similar size were analyzed for a total of 20 sections. For Otx $1^{\text {cre/+ }}$; Otx $2^{+/-}$control embryos, an identical rectangular region with the major side corresponding to the neuroepithelial border facing the ventricle of the thalamus was selected. Three sections per each control embryo for a total of five embryos were in the thalamus, in which this is reflected in a mosaic-like inactivation of Otx2 first detected at E10.5.

\section{Anatomical and histological analysis of the thalamus}

$O t x 1^{\text {cre/+ }}$; Ot $x 2^{\text {flox/- }}$ mice died at birth (Puelles et al., 2003). Morphological analysis of the thalamus of conditional mutants showed no obvious impairments before E11 (data not shown), although severe abnormalities characterized by the presence of invaginations and swellings of the neuroepithelium were revealed at E12.5 (Fig. 2B). At E13.5, cell masses of irregular size were numerous, and some of them reached a remarkable size, exhibited a ventricle-like cavity, and resembled patches of proliferating neuroepithelium (Fig. 2D). At late gestation, cell masses were 


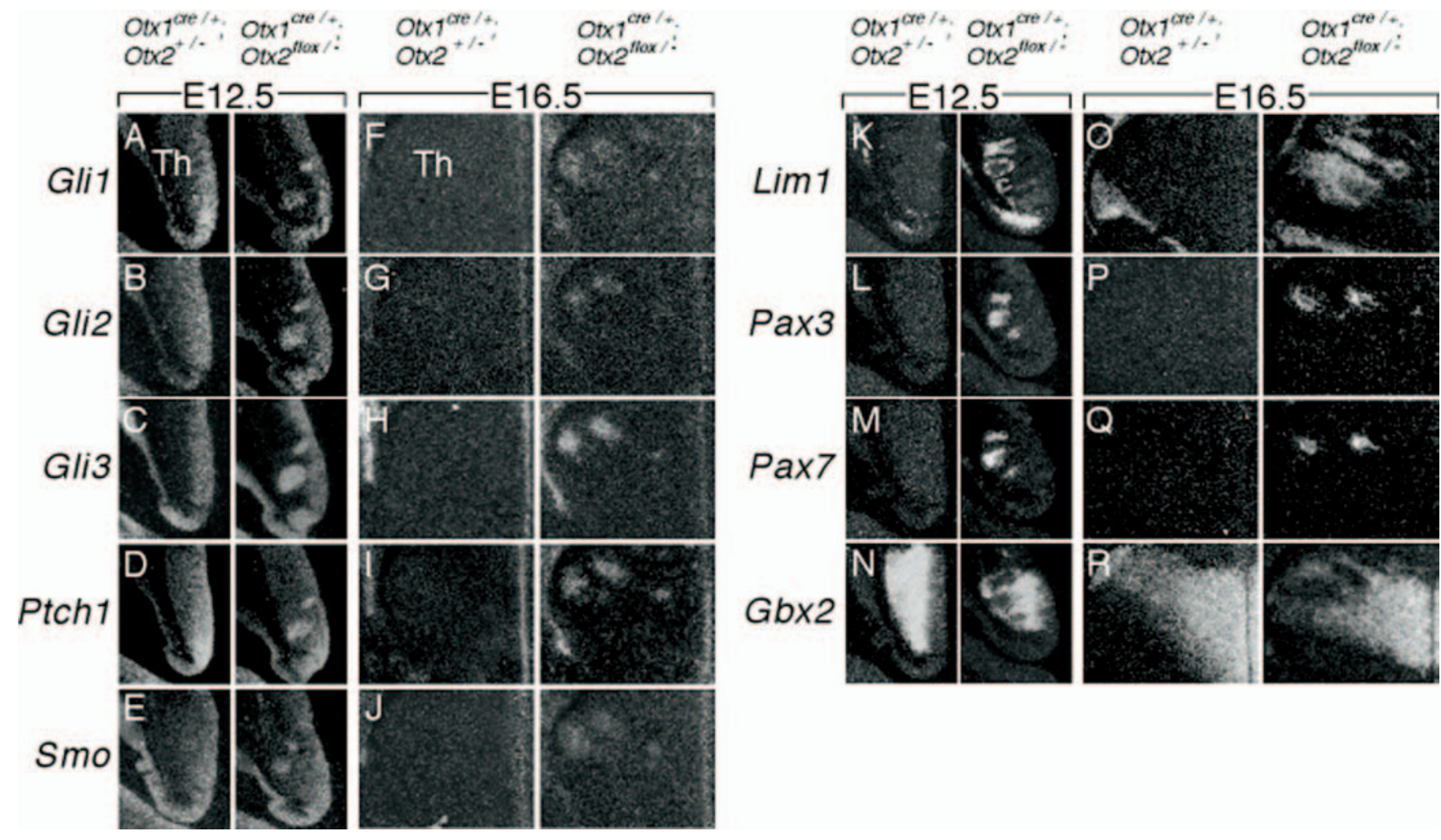

Figure 3. Abnormal gene expression in the patches. $\boldsymbol{A}-\boldsymbol{R}$, In situ hybridization of E12.5 and E16.5 0 tx $7^{\text {cre/ } /+} ; 0 t \times 2^{+/-}$and conditional mutants with $\operatorname{Gli1}(\boldsymbol{A}, \boldsymbol{F}), \boldsymbol{G l i 2}(\boldsymbol{B}, \boldsymbol{G}), \boldsymbol{G l i 3}(\boldsymbol{C}, \boldsymbol{H}), \operatorname{Ptch} 1(\boldsymbol{D}$, I), Smo $(\boldsymbol{E}, \boldsymbol{J}), \operatorname{Lim} 1(\boldsymbol{K}, \mathbf{O}), \operatorname{Pax} 3(\boldsymbol{L}, \boldsymbol{P}), \operatorname{Pax} 7(\boldsymbol{M}, \mathbf{Q})$, and $G b \times 2(\boldsymbol{N}, \boldsymbol{R})$ probes. Note that $G b \times 2$ is lost in the patches in which the expression of Lim1, Pax3, and Pax7 is activated. For control embryos, the sections at $E 12.5$ from $\boldsymbol{A}-\boldsymbol{D}$ and from $\boldsymbol{E}-\boldsymbol{N}$ are two different groups of adjacent sections; similarly, also for the conditional mutant, the sections at E12.5 from $\boldsymbol{A}-\boldsymbol{D}$ and from $\boldsymbol{E}-\boldsymbol{N}$ are two groups of adjacent sections belonging to two different embryos. Th, Thalamus.

randomly distributed within the mantle zone of the thalamus (Fig. $2 F$ ), where the routes of axonal tracts were heavily affected as revealed by neurofilament staining (Fig. $2 H$ ). This abnormality may account for the lethality of conditional mutants. None of these impairments was detected in $\mathrm{Otx} \mathrm{I}^{\mathrm{cre} /+}$; Otx $2^{+/-}$control embryos (Fig. 2). We also noted that these cell masses exhibited a size variability quite pronounced. To better describe this aspect, we studied in detail E16.5 Otx $1^{\text {cre/+ }}$; Otx $2^{f l o x /-}$ mutants. Approximately $20 \%$ of cell masses scored at E16.5 belonged to the group with large size (Fig. $2 F$ ). In the same embryos, the majority of the cell masses $(\sim 55 \%)$ was of intermediate size, which means in our arbitrary scale between one-half and one-fourth of those with large size. The residual patches $(\sim 25 \%)$ detected in these embryos showed a small size, corresponding to one-half or less of those classified as intermediate in size (Fig. $2 F$ ). In the thalamus of the E16.5 scored embryos, we detected in total 14-28 cell masses. Regarding the location, cell masses detected at E12.5 and E13.5 were identified within the subventricular zone or at the boundary between the subventricular and the mantle zone, whereas those of large size were prevalently detected in the mantle zone (Fig. $2 B, D$ ). At E16.5, we only sporadically detected cell masses close to the thalamic ventricular neuroepithelium, with the large majority of the patches located in the mantle zone (Fig. $2 F)$. Finally, we also noted that large patches are sometimes generated by the fusion of multiple cell masses. We interpreted this heterogeneity in size and location as a consequence of the extent and stage of Otx2 inactivation. Thus, large cell masses may reflect an early inactivation of $\mathrm{Otx2}$ (e.g., at E10.5) in a relatively large number of progenitors, whereas small cell masses close to the ventricle may be caused by a late inactivation (e.g., E12.5 and/or E13.5) of Otx2 in few progenitors.

\section{Lack of Otx 2 affects cell identity}

To investigate whether the lack of Otx2 generates abnormalities in cell identity, we first analyzed the expression of a number of molecular markers at E12.5 and E16.5. These included components of the Shh pathway that are normally expressed in the proliferating neuroepithelium of the thalamus such as Gli1, Gli2, Gli3, Smo, and Ptch1, and genes transcribed in territories adjacent to the thalamus, such as Pax3, Pax7, and $\operatorname{Lim} 1$ in the pretectum, Dlx1 and Dlx5 in the prethalamus, or expressed in the mantle zone of the thalamus such as Gbx2. Moreover, we monitored the expression of Isll, Foxa2 [also known as $H n f 3 \beta$ (hepatocyte nuclear factor)], Msxi, three members of the $N k x$ gene family such as $N k \times 2.1, N k \times 2.2$, and $N k x 6.1$, two pallial markers such as Tbr1 and Emxl, and genes encoding signaling molecules such as Shh, Fgf8, Wnt1, Wnt3a, Bmp2, and Bmp6. At E12.5 and E16.5 in conditional mutants, no expression was detected in the patches for Dlx1, Dlx5, Tbr1, Emx1, Msx1, Foxa2, Isl1, and Nkx genes or any of the signaling molecules mentioned above (data not shown). For example, Shh was normally expressed in the forebrain and, in particular, its expression in correspondence of the zona limitans intrathalamica (zli) was unaffected (data not shown). However, Gli1, Gli2, Gli3, Ptch1, and Smo were transcribed in both the unaffected neuroepithelium and the patches (Fig. $3 A-J$ ), suggesting that the patches contain cells sharing similarity with progenitors normally located in the subventricular and ventricular zones.

Interestingly, abundant expression of Lim1, Pax3, and Pax7 and a complementary loss of $G b \times 2$ was detected in the patches (Fig. $3 K-R$ ). The abnormal expression of these genes was detected reproducibly in all of the patches of conditional mutants analyzed and never in $\mathrm{Otx} \mathrm{Cre/+}^{\mathrm{cr}} ; \mathrm{Otx} 2^{+/-}$control embryos (Fig. 


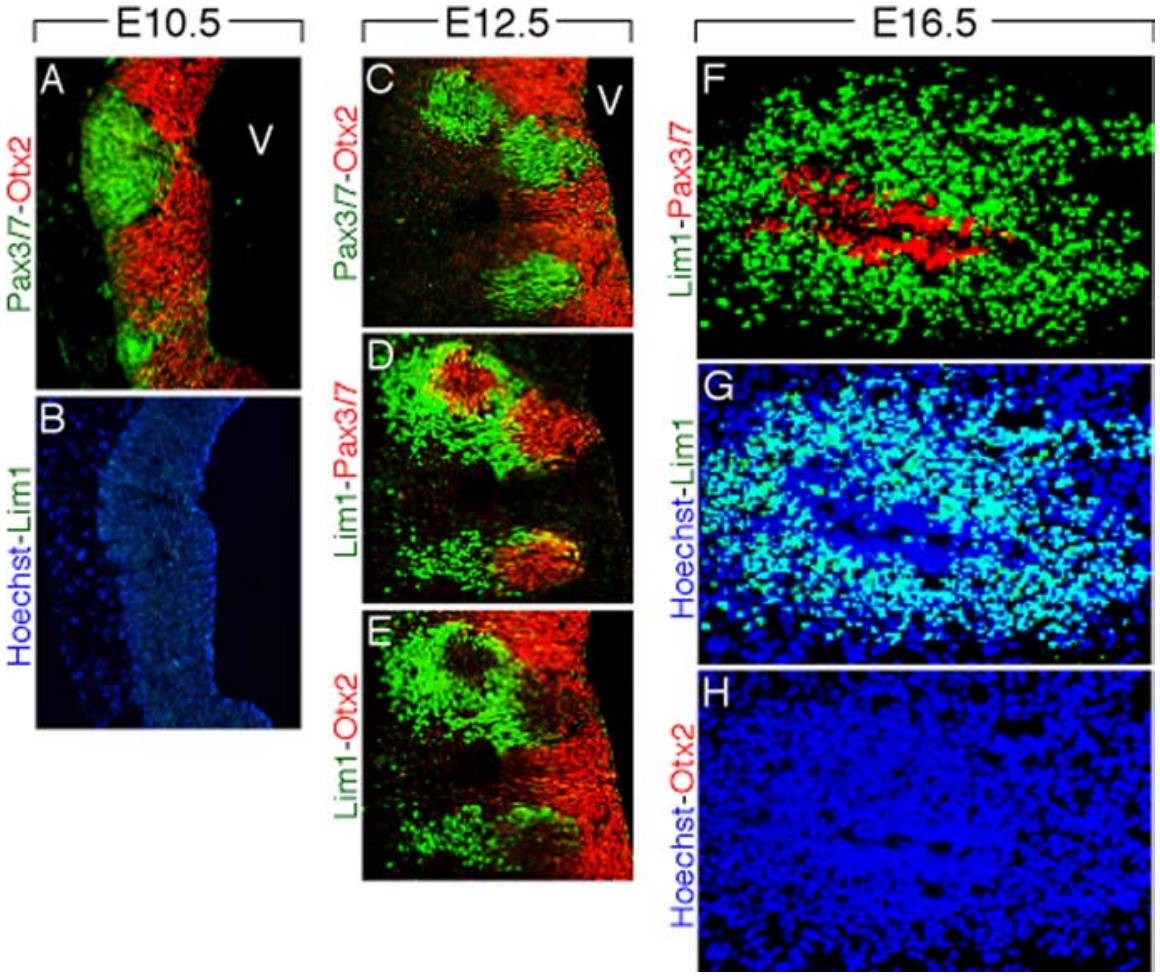

Figure 4. Cell-type identity in the patches. $\boldsymbol{A}-\boldsymbol{H}$, Combinatorial immunodetection with $0 \operatorname{tx2}(\boldsymbol{A}, \boldsymbol{C}, \boldsymbol{E}, \boldsymbol{H}), \operatorname{Pax} 3 / 7(\boldsymbol{A}, \boldsymbol{C}, \boldsymbol{D}, \boldsymbol{F})$ and $\operatorname{Lim} 1(\boldsymbol{B}, \boldsymbol{D}, \boldsymbol{E}-\boldsymbol{G})$ antibodies. The sections at E10.5 and E16.5 are counterstained with Hoechst. The red color for $\mathrm{Pax} 3 / 7(\boldsymbol{D})$ is a pseudocolor. V, Ventricle.

$3 K-R)$. Thus, the lack of Otx2 generates dramatic transcriptional abnormalities in the patches such as the activation of genes not normally transcribed in the thalamus and the repression of $G b \times 2$, normally transcribed in the mantle zone of the thalamus. Noteworthy, Lim1, Pax3, and Pax7 are normally coexpressed in the pretectum (data not shown) (supplemental Fig. S1, available at www.jneurosci.org as supplemental material).

\section{Otx2 is required to suppress the generation of multiple cell types}

To assess the molecular identity at the single-cell level and the timing of cell-type generation in the patches, a combined analysis of the distribution of Otx2, Pax3, Pax7, and Lim1 proteins was performed at E10.5, E12.5, E13.5, and E16.5. To this aim, we developed a procedure allowing the comparison of several antibodies on the same section to attribute a complex molecular code, if any, to the same cell (see Materials and Methods). For Pax3 and Pax7, we used an antibody recognizing both proteins because two antibodies specifically directed against Pax3 or Pax7 showed full colocalization (data not shown). At E10.5, all of the progenitors lacking Otx2 exhibited a strong activation of Pax3/7 (Fig. 4A), whereas no expression was detected for Lim1 (Fig. 4B).

At E12.5 and E13.5 (data not shown), $\mathrm{Lim}^{+}$cells were detected preferentially on the lateral edge of the Pax $3 / 7^{+}$-Otx2 ${ }^{-}$ domain (Fig. 4C-E). A few of these were also Pax3/7 ${ }^{+}$(Fig. 4D), suggesting that these cells may represent a transitory population of $\mathrm{Pax} 3 / 7^{+}$-Otx $2^{-}$differentiating precursors (see below). At E16.5, the patches reached a remarkable size, and, as at the earlier stages, the Pax $3 / 7^{+}$cells were restricted to the inner region of the patches (Fig. $4 F$ ) whereas Lim $1^{+}$cells were distributed in the outer region of the patches (Fig. $4 F, G$ ). Then, we studied whether qualitative variations were detected in the patches for Pax $3 / 7$ and
Lim1 expression. To this aim, we analyzed 115 patches at E12.5 and 105 at E16.5. In all of these patches, the same pattern of gene expression reported in Figure 4 was revealed. This meant that $\mathrm{Pax} 3 / 7^{+}$cells always corresponded to the Otx $2^{-}$progenitors and that Lim $1^{+}$cells were always detected at the edge of the Pax $3 / 7^{+}$-Otx $2^{-}$ cells. Moreover, a numerical comparison between $\mathrm{Pax} 3 / 7^{+}$-Otx $2^{-}$and $\mathrm{Lim} 1^{+}$cells, performed on 80 patches at E12.5 and 80 at E16.5, revealed that the number of $\mathrm{Pax} 3 /$ $7^{+}$-Otx2 ${ }^{-}$cells was between 25 and $45 \%$ of the Lim $1^{+}$cells at E12.5 and between 10 and $25 \%$ at E16.5. Noteworthy, in $10 \%$ $(n=8)$ of the patches analyzed at E12.5 and in $6 \%(n=5)$ of those scored at E16.5, this proportion between $\mathrm{Pax} 3 / 7^{+}$-Otx $2^{-}$ and $\mathrm{Lim}^{+}$cells changed to $\sim 65 \%$ at E12.5 and $40 \%$ at E16.5.

In summary, these data indicate that lack of Otx 2 in a restricted number of progenitors of the thalamus affects their normal identity, as revealed by the early derepression of $\mathrm{Pax} 3 / 7$, and is subsequently reflected in the appearance of Lim $1^{+}$cells.

\section{Proliferation and cell fate analysis}

Previous experiments did not prove whether (1) Pax $3 / 7^{+}$-Otx2 ${ }^{-}$cells corresponded to proliferating progenitors, (2) proliferation in the patches was abnormal, and (3) Lim ${ }^{+}$cells represented the postmitotic progeny of the $\mathrm{Pax} 3 / 7^{+}-\mathrm{Otx} 2^{-}$ precursors.

To address these issues, we first studied cell proliferation by providing a short pulse $(60 \mathrm{~min})$ of BrdU to pregnant females at E10.5, E12.5, E13.5, and E16.5.

In wild-type (data not shown) and Ot $x 1^{\text {cre/+}} ;$ Ot $x 2^{+/-}$control embryos, BrdU ${ }^{+}$cells were detected at E10.5 throughout the entire neuroepithelium and, subsequently, within the ventricular and subventricular zones, in which they gradually decreased in number and became sporadic at E16.5 (Fig. 5A-D). At E10.5, no obvious difference in the number of $\mathrm{BrdU}^{+}$cells was observed between the $\mathrm{Pax} 3 / 7^{+}-\mathrm{Otx} 2^{-}$and the unaffected neuroepithelium of conditional mutants or control embryos (Fig. $5 A, I, M$ ); conversely, at E12.5 and particularly at E13.5 the number and density of $\mathrm{BrdU}{ }^{+}$cells within the $\mathrm{Pax} 3 / 7^{+}-\mathrm{Otx} 2^{-}$patches was remarkably increased (Fig. $5 \mathrm{~J}, \mathrm{~K}, \mathrm{~N}, \mathrm{O}$ ) when compared with the unaffected adjacent neuroepithelium (Fig. $5 J, K, N, O$, yellow arrow) or with control embryos (Fig. $5 B, C$ ). At E16.5, the number of $\mathrm{BrdU}^{+}$cells diminished but appeared reproducibly higher in the patches of conditional mutants (Fig. $5 L, P$ ). Noteworthy, between $\mathrm{E} 12$ and $\mathrm{E} 14$, the size of $\mathrm{BrdU}^{+}-\mathrm{Pax} 3 / 7^{+}$cell masses greatly expanded, sometimes reaching the lateralmost edge of the thalamus (Fig. $5 J, K$ ).

Next, to assess whether the increased number of precursors in $S$ phase was reflected in a corresponding increase in mitotic activity, we determined the number of $\mathrm{Ph}-\mathrm{H} 3$-positive cells. In conditional mutants at $\mathrm{E} 10.5, \mathrm{Ph}-\mathrm{H} 3{ }^{+}$cells were detected in the Pax $3 / 7^{+}$patches (Fig. 5Q, white arrows), and, as for the $\mathrm{BrdU}^{+}$ cells, their number and density were approximately comparable with those of control embryos (Fig. 5E) or the unaffected neuroepithelium of conditional mutants (Fig. 5Q, yellow arrows). 
However, at E10.5, the position of $\mathrm{Ph}-\mathrm{H} 3^{+}$ cells was abnormal in correspondence of $\operatorname{Pax} 3 / 7^{+}$patches, suggesting an early alteration in the proliferation pattern. At E12.5 and E13.5, the number of Ph-H3 ${ }^{+}$cells was clearly increased along the ventricle-like cavity of the patches (Fig. 5F, G and compare yellow and white arrows in $R, S$ ). At E16.5, the number of $\mathrm{Ph}-\mathrm{H} 3{ }^{+}$cells decreased but was still reproducibly higher in the patches (Fig. 5, compare $H, T$ ).

Cell counting at E13.5 (see Materials and Methods) showed that, compared with Otx $1^{\text {cre/+ }}$; Ot $x 2^{+/-}$control embryos, the number of $\mathrm{BrdU}^{+}$and $\mathrm{Ph}-\mathrm{H} 3{ }^{+}$cells detected in the patches was increased by 1.8- and 1.7-fold, respectively, whereas the ratio between $\mathrm{Ph}-\mathrm{H}_{3}{ }^{+}$and $\mathrm{BrdU}^{+}$cells in control embryos (0.11) and in the patches (0.12) was comparable. This indicates that cell cycle progression from the $S$ to the $M$ phase is not impaired.

Therefore, these findings collectively support the possibility that Otx2, directly or indirectly, controls the number of proliferating progenitors by regulating their postmitotic transition between E12 and E14. Failure in this control may result in an increase of Otx $2^{-}$progenitors reentering the cell cycle and may account for local overgrowth generating neuroepithelial invaginations and the subsequent incorporation of cell masses within the mantle zone.

To assess whether Lim $1^{+}$neurons represented the postmitotic progeny of Pax3/ $7^{+}$-Otx2 ${ }^{-}$progenitors, we first studied whether $\mathrm{Lim}^{+}$cells were also $\mathrm{BrdU}^{+}$in short-pulse experiments. In these experiments, Lim1 ${ }^{+}$cells did not show BrdU staining (data not shown), thus indicating that they correspond to postmitotic neurons. Then, we performed a birthdating experiment by providing a long pulse of BrdU at E13.3 (see Materials and Methods) to pregnant females and analyzing at E16.5 whether postmitotic neurons BrdU labeled at E13.3 expressed Lim1. In this experiment, $\mathrm{BrdU}^{+}$cells appeared at E16.5 homogeneously distributed in a ring-like shape in the patches and, importantly, did not mix with BrdU ${ }^{+}$cells generated in the unaffected ventricular zone (Fig. 5U). Coimmunostaining experiments showed that a relevant number of $\operatorname{Lim} 1^{+}$postmitotic neurons were also $\mathrm{BrdU}^{+}$(Fig. 5V,W), indicating that Pax3/ $7^{+}$-Otx $2^{-}$progenitors generate $\mathrm{Lim} 1^{+}$ postmitotic neuronal cell types. Moreover, because scattered $\mathrm{BrdU}^{+}$-Lim1 ${ }^{-}$cells were reproducibly observed (Fig. $5 \mathrm{~V}, \mathrm{~W}$, arrowheads), it is conceivable that these cells represent an additional population of postmitotic neurons unidentified by our markers and generated by the Pax $3 / 7^{+}$-Otx $2^{-}$progenitors.

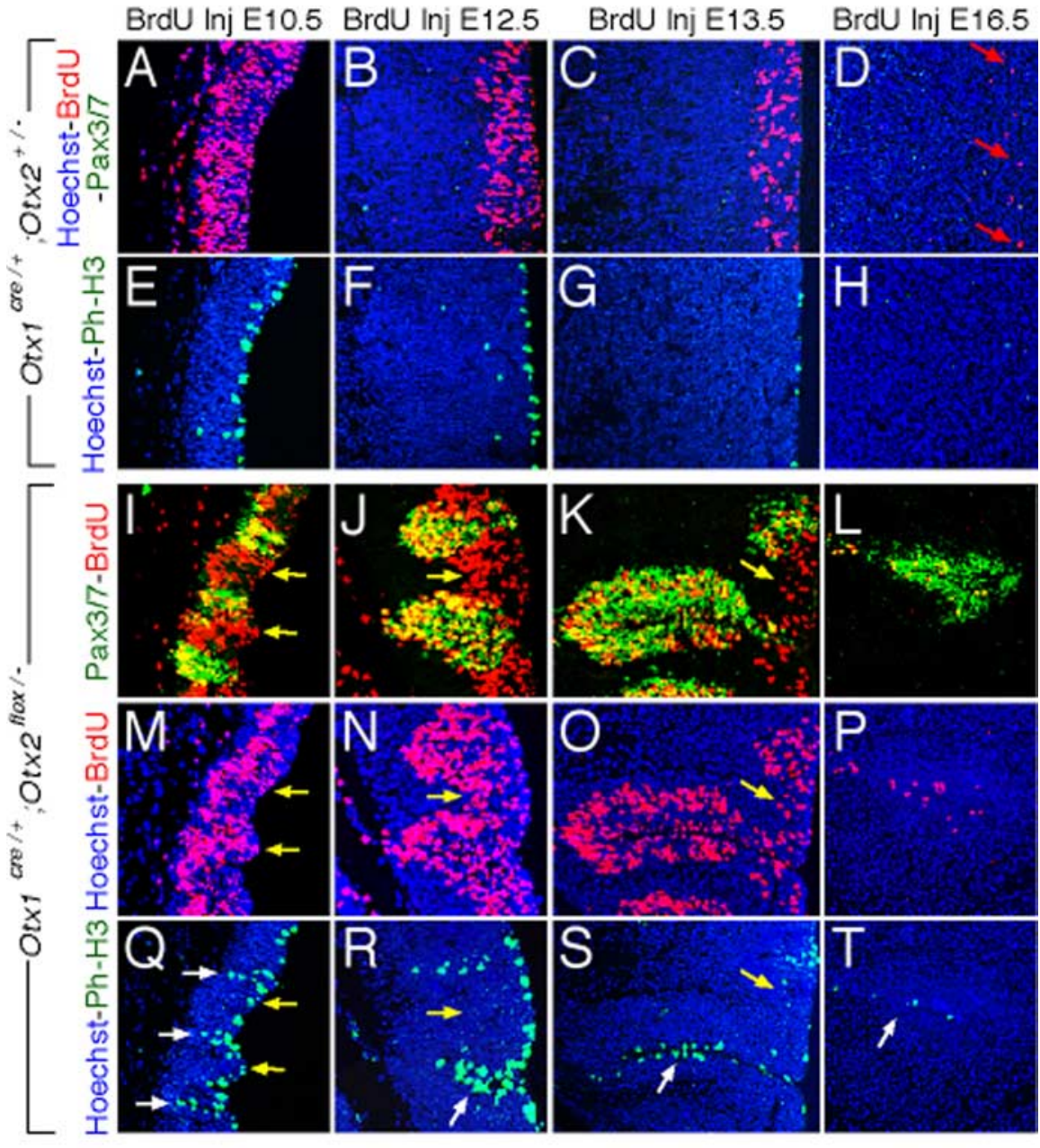

Ot $\times 1^{\mathrm{cre} / \mathrm{t}} ;$ Ot $\times 2^{\text {flox/. }}$

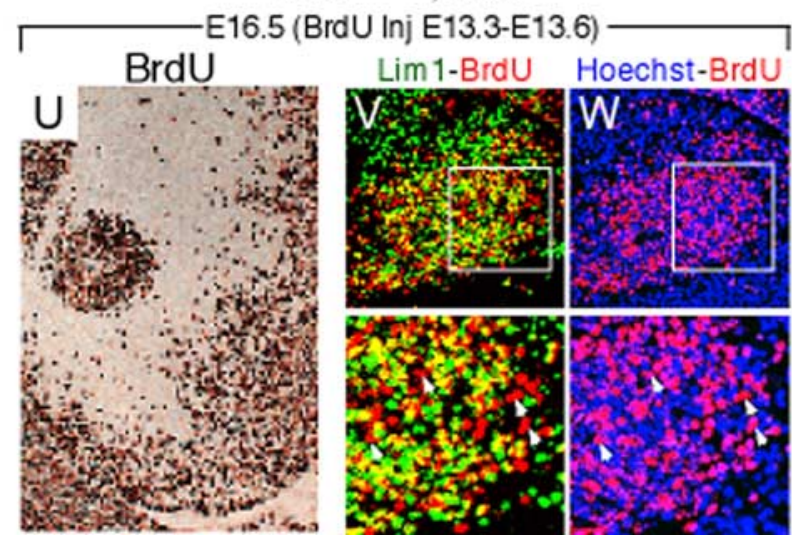

Figure 5. Proliferating activity and cell fate analysis. $\boldsymbol{A}-\boldsymbol{T}$, Immunodetection of $\mathrm{Pax} 3 / 7$ and $\operatorname{BrdU}(\boldsymbol{A}-\boldsymbol{D}, \boldsymbol{I}-\boldsymbol{L}), \mathrm{BrdU}$ alone $(\boldsymbol{M}-\boldsymbol{P})$, and Ph-H3 $(\boldsymbol{E}-\boldsymbol{H}, \mathbf{Q}-\boldsymbol{T})$ in embryos exposed to a short pulse of BrdU at E10.5, E12.5, E13.5, and E16.5. Note that, at E12.5 and particularly at $\mathrm{E} 13.5$, the density of BrdU ${ }^{+}$and $\mathrm{Ph}-\mathrm{H} 3{ }^{+}$cells within the patches $(J, K, N, \mathbf{O}, R, S)$ is remarkably increased when compared with the unaffected neuroepithelium of conditional mutants (yellow arrow in $J, K, N, \mathbf{O}, \boldsymbol{R}, \boldsymbol{S}$ ) or $0 \operatorname{txx}^{7^{\text {re } /+}} ; 0 \mathrm{tx} 2^{+/-}$ control embryos $(\boldsymbol{B}, \boldsymbol{C}, \boldsymbol{F}, \boldsymbol{G})$. The red arrows in $\boldsymbol{D}$ point to the sporadic BrdU ${ }^{+}$cells detected at E16.5 in control embryos, and the white arrows in Q- $\boldsymbol{T}$ point to the $\mathrm{Ph}-\mathrm{H} 3^{+}$cells in the patches. $\boldsymbol{U}-\boldsymbol{W}$, BrdU long-pulse experiments show that BrdU ${ }^{+}$cells labeled between E13.3 and E13.6 accumulate at E16.5 in the patches without mixing with those labeled along the unaffected neuroepithelium $(\boldsymbol{U})$, and most of them are $\operatorname{Lim}^{+}(\boldsymbol{V})$. Note that BrdU ${ }^{+}$-Lim1 ${ }^{-}$cells (arrowheads in the magnifications) may correspond to additional cell type(s) of the patches $(\boldsymbol{V}, \boldsymbol{W})$.

\section{Lack of Otx 2 induces GABAergic differentiation in} glutamatergic progenitors

We next analyzed whether the lack of Otx 2 caused abnormalities in terminal differentiation. 

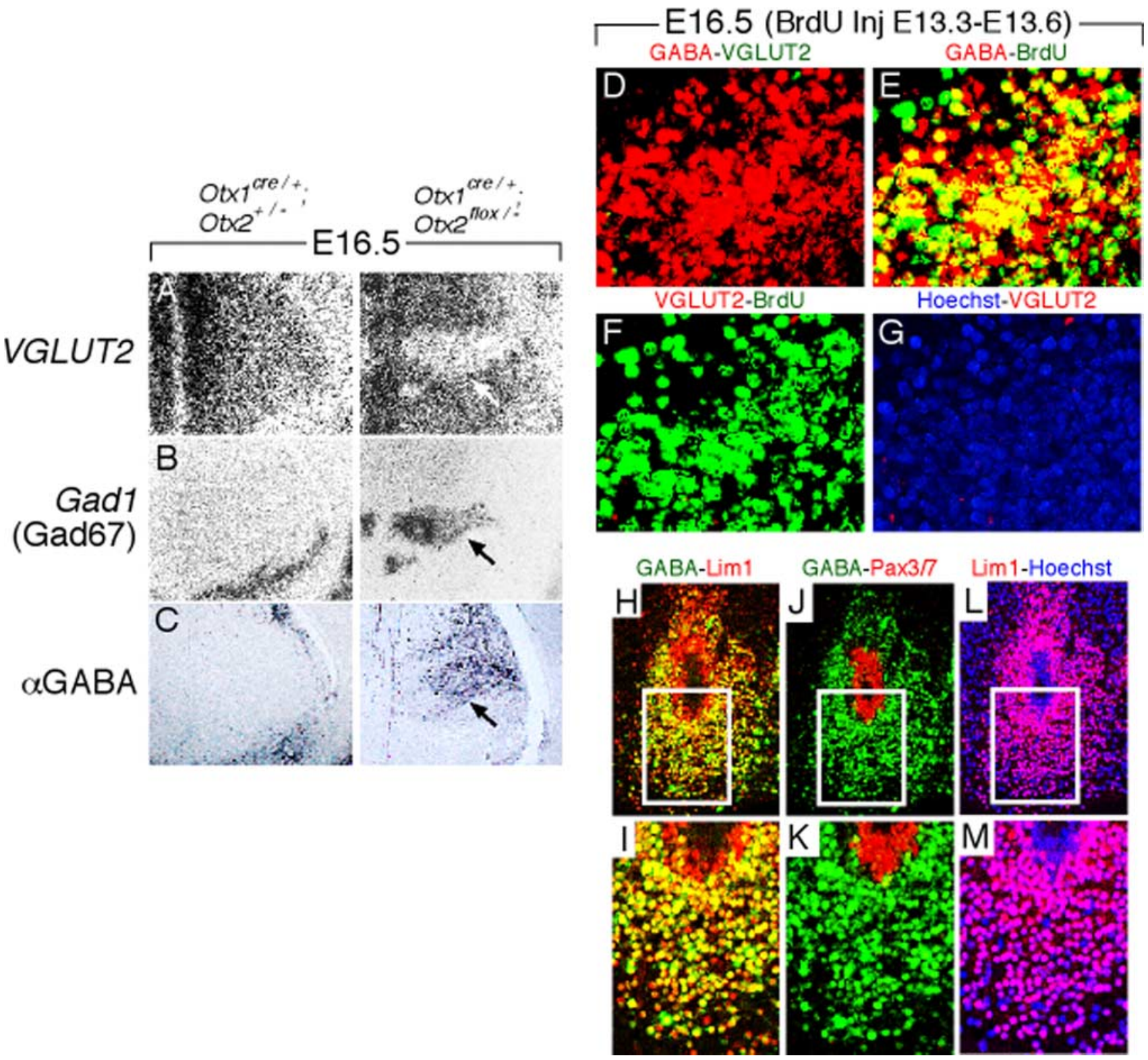

Figure 6. GABAergic fate switch of glutamatergic progenitors. $A-C$, In situ hybridization $(A, B)$ and immunohistochemistry $(\boldsymbol{C})$ of $\mathrm{E} 16.5$ Otx $1^{\text {cre/+}} ;$ Otx $2^{+/-}$and 0 tx $7^{\text {cre/+}} ; 0$ tx $2^{\text {flox } /-}$ embryos with VGLUT2 $(\boldsymbol{A})$, Gad1 $(\boldsymbol{B})$ probes, and GABA $(\boldsymbol{C})$ antibody show that, in the patches, the lack of VGLUT2 expression correlates with the induction of GABAergic markers. $D-G$, Combinatorial immunodetection with GABA $(\boldsymbol{D}, \boldsymbol{E}), \operatorname{VGLUT2}(\boldsymbol{D}, \boldsymbol{F}, \boldsymbol{G})$, and $\operatorname{BrdU}(\boldsymbol{E}, \boldsymbol{F})$ antibodies in $\mathrm{E} 16.5$ conditional mutants labeled at E13.3 with BrdU shows that many of the BrdU ${ }^{+}$neurons are $\mathrm{GABA}{ }^{+}$, although none of them are VGLUT2 ${ }^{+}$. $\boldsymbol{H}-\boldsymbol{M}$, Combinatorial immunodetection with GABA $(\boldsymbol{H}-\boldsymbol{K}), \operatorname{Lim} 1(\boldsymbol{H}, \boldsymbol{I}, \boldsymbol{L}, \boldsymbol{M})$, and Pax3/7 $(\boldsymbol{J}, \boldsymbol{K})$ antibodies shows that GABA ${ }^{+}$neurons are $\operatorname{Lim} 1^{+} . \boldsymbol{I}, \boldsymbol{K}$, and $\boldsymbol{M}$ correspond to the magnification of the area demarcated in $\boldsymbol{H}, \boldsymbol{J}$, and $\boldsymbol{L}$. The green color for VGLUT2 (D) is a pseudocolor.

Because the thalamus is a relevant source of glutamatergic neurons, we first tested whether this major fate was retained in the patches by studying the expression of the vesicular glutamate transporter VGLUT2 (Cheng et al., 2004; Fremeau et al., 2004; Schuurmans et al., 2004). In contrast to control embryos, VGLUT2 transcripts were not detected in the patches (Fig. 6A, arrow).

Then we studied whether the lack of Otx2 was reflected in a GABAergic fate switch by using specific markers such as glutamic acid decarboxylase Gad1 (encoding GAD67) and GABA. Strikingly, abundant transcription of Gad1 and numerous $\mathrm{GABA}^{+}$ cells were identified in the patches (Fig. $6 B, C$, arrow). Detailed inspection at the single-cell level, in E13.5 (data not shown) and E16.5 embryos, showed that all of the $\mathrm{GABA}^{+}$cells were VGLUT2 $^{-}$(Fig. 6D). Moreover, to reinforce this finding and to examine whether $\mathrm{GABA}^{+}$cells corresponded to postmitotic neurons belonging to the patches, we performed BrdU long-pulse experiments similar to those shown previously for Lim1 (Fig. 5). In this way, we assessed whether at E16.5 $\mathrm{BrdU}^{+}$cells labeled at E13.3 expressed GABA and/or VGLUT2. This experiment showed that a relevant percentage of $\mathrm{BrdU}^{+}-\mathrm{GABA}^{+}$neurons was identified (Fig. 6E), whereas no neurons coexpressing VGLUT2 and BrdU were detected (Fig. $6 F, G$ ). This indicates that glutamatergic fate is totally abolished in the postmitotic progeny of the Otx2 $2^{-}$progenitors. Comparison of $\mathrm{GABA}^{+}$neurons with those expressing $\operatorname{Lim} 1$ showed that all of the $\mathrm{GABA}^{+}$cells were Lim $1^{+}$(Fig. $6 \mathrm{H}, I$ ), and Pax $3 / 7^{+}$progenitors were $\mathrm{GABA}^{-}$(Fig. $6 J, K)$.

To investigate whether postmitotic cells in the patches expressed markers of additional cell fates, we analyzed the distribu- 
tion of $\mathrm{DBH}$ and $\mathrm{TH}$ for catecholaminergic fate and 5-HT for serotoninergic fate. In these experiments, no expression for these markers was detected.

These data indicate that Otx2 is required in glutamatergic progenitors of the thalamus to suppress the GABAergic differentiation program. In this context, the finding that all of the GABAergic neurons are Lim ${ }^{+}$suggests that Lim1 might represent a postmitotic selector of the GABAergic differentiation program. Altogether, our data also indicate that all of the abnormalities so far described in the patches are compatible with a cell-autonomous requirement for Otx2.

Lack of Otx2 induces Mash1 expression In the forebrain, the proneural genes $\mathrm{Ngn} 1$, Ngn2, and Mash1 are expressed in different pools of progenitors in which they are required for neuronal specification. In the dorsal telencephalon and thalamus, Ngns, in particular Ngn2, play a dual role, both being required to activate glutamatergic differentiation of early born neurons and to repress Mash 1 expression and GABAergic fate (Fode et al., 2000; Schuurmans et al., 2004). We therefore studied the expression of Mash1 and Ngn2 in the patches.

Robust expression of Mash1 was first detected in a relevant fraction of the Pax $3 / 7^{+}$progenitors at E10.5 and, subsequently, maintained until late development (Fig. 7A-C). Importantly, most but not all of the Pax $3 / 7^{+}$-Otx $2^{-}$progenitors exhibited Mash1 expression, thus suggesting that the total absence of glutama-

tergic neurons (Fig. 6) should primarily depend on the lack of Otx2 in all of the progenitors rather than on the activation of Mash1 in only a percentage of them. In this context, the activation of $\mathrm{Pax} 3 / 7$ in all of the Otx $2^{-}$progenitors strongly suggests that lack of glutamatergic fate may be the consequence of an early and general change in the identity of the mutant progenitors.

Next, we analyzed whether the activation of Mash 1 correlated with the suppression of Ngn2. We found that, at E10.5, Ngn2 exhibited along the thalamic neuroepithelium of control and conditional mutant embryos the same expression pattern (Fig. $7 D, G$ and data not shown). In particular, in correspondence of the patches, Ngn2 was detected in both Mash1 ${ }^{+}$(Fig. 7D) and Pax $3 / 7^{+}$(Fig. $7 G$ ) progenitors but, subsequently, its expression was gradually reduced and totally lost at E16.5 (Fig. 7 E, F, H, I). These findings suggest that, in the absence of Otx2, (1) Ngn2 expression is not affected until E12 and is subsequently lost, and (2) Ngn2 is not sufficient to repress Mash1. In this context, the gradual loss of Ngn2 expression may reflect GABAergic fate switch of Otx $2^{-}$progenitors.

\section{Discussion}

Otx 2 controls identity and fate of glutamatergic progenitors in the thalamus

Otx genes are required for regionalization and patterning of the developing brain and, in particular, play a crucial role in the

\section{Otx $1^{\text {cre } /+} ; O t \times 2^{\text {fiox/. }}$}

E12.5
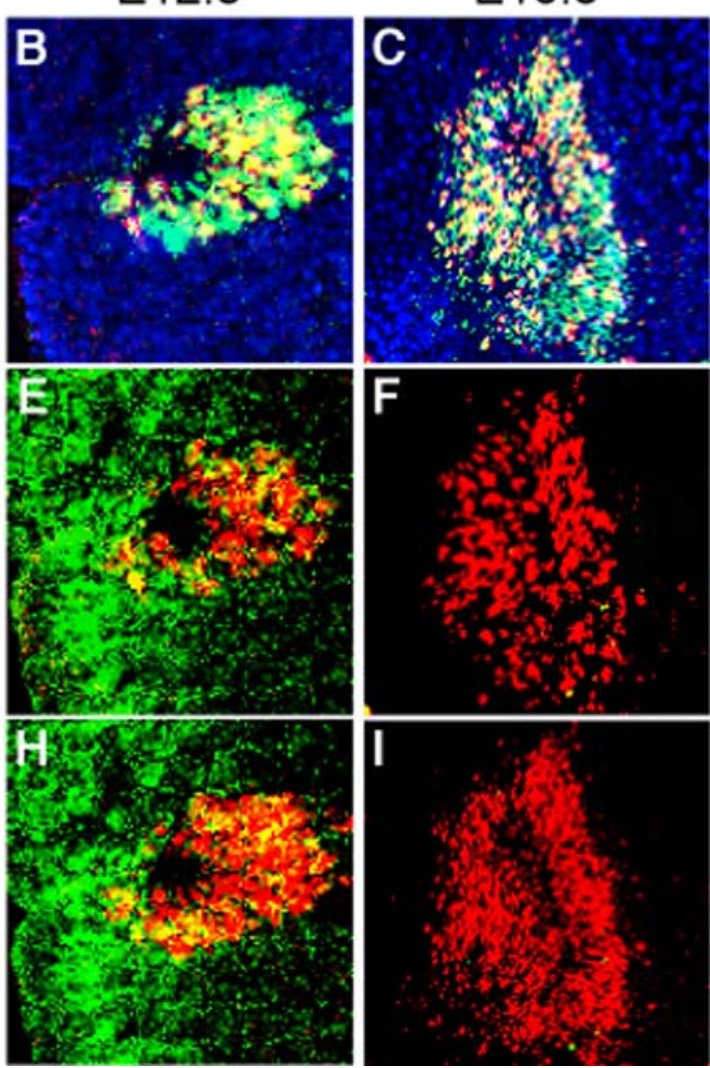

Figure 7. Activation of Mash1 in $0 \mathrm{tx} 2^{-}-\mathrm{Pax} 3 / 7^{+}$progenitors and $0 \mathrm{tx2} / \mathrm{Ngn} 2$ synergistic cooperation. $A-I$, Combinatorial immunodetection with Mash1 $(\boldsymbol{A}-\boldsymbol{F}), \operatorname{Pax3} / 7(\boldsymbol{A}-\boldsymbol{C}, \mathbf{G}-\boldsymbol{I})$, and $\mathrm{Ngn} 2(\boldsymbol{D}-\boldsymbol{I})$ shows that, at $\mathrm{E} 10.5$, Mash1 is activated in a relevant fraction of Pax $3 / 7^{+}$cells $(\boldsymbol{A})$ and maintained up to late gestation $(\boldsymbol{B}, \boldsymbol{C})$, whereas Ngn2 expression is unaffected at $E 10.5(\boldsymbol{D}, \boldsymbol{G})$ and then gradually lost $(\boldsymbol{E}, \boldsymbol{F}, \boldsymbol{H}, \boldsymbol{I})$. The green color for $\operatorname{Pax} 3 / 7(\boldsymbol{A}-\boldsymbol{C})$ is a pseudocolor.

establishment of the identity and fate of progenitors located in the ventral midbrain (Puelles et al., 2003, 2004).

As for other regulatory factors, Otx2 is expressed in different brain areas generating specific neuronal cell types. Therefore, understanding how the same protein controls identity and fate of different populations of progenitors may provide relevant insights into the mechanisms by which neuronal diversity is generated.

Here we studied the role of Otx 2 in the thalamus to determine whether it is required for the differentiation of glutamatergic progenitors and the regulatory steps in which it is involved.

Our results indicate the following: (1) Otx2 is required for glutamatergic differentiation; (2) lack of Otx2 and GABAergic fate switch correlate with the activation of Mash1 in progenitors expressing Ngn2; (3) lack of Otx2 also correlates with the activation of a pretectal-like gene expression profile as revealed by the induction of Pax3, Pax7, and Lim1; and (4) Otx2 has a relevant role in the control of proliferation. Together, these findings indicate that, in the thalamus, Otx2 is a novel and crucial molecular determinant controlling identity, fate, and proliferation of glutamatergic progenitors.

Relevant data have been provided previously on the genetic functions controlling GABAergic and glutamatergic differentiation (Casarosa et al., 1999; Fode et al., 2000; Marin and Rubenstein, 2002; Parras et al., 2002; Cheng et al., 2004; Miyoshi et al., 2004; Schuurmans et al., 2004). Among these, it has been shown 
that, in the telencephalon and thalamus, Ngns, in particular Ngn2, are required to promote glutamatergic fate and to simultaneously repress Mash1 and GABAergic differentiation (Fode et al., 2000; Schuurmans et al., 2004). Conversely, in the basal forebrain, Ngn2 is unable to repress Mash1 expression (Parras et al., 2002). Our results show that, in early thalamic progenitors (E10.5) lacking Otx2, glutamatergic differentiation is abolished and Mash1 is de-repressed in a relevant subset of Otx $2^{-}-\mathrm{Pax} 3 /$ $7^{+}$-Ngn2 ${ }^{+}$progenitors, suggesting that Ngn2 is not sufficient to suppress Mash1 activation in an Otx $2^{-}$cellular context. Therefore, our findings together with those reported previously (Fode et al., 2000; Parras et al., 2002; Schuurmans et al., 2004) suggest that lack of Otx2 or Ngn2 in the thalamus generates in both cases Mash1 de-repression and GABAergic fate switch. Noteworthy, in the forebrain areas in which Otx2 and Ngns are coexpressed (e.g., dorsal telencephalon and thalamus), Mash1 transcription and GABAergic fate are repressed, whereas glutamatergic differentiation is activated; the opposite occurs in the areas in which only Otx2 (e.g., pretectum) or none of them (e.g., basal telencephalon and prethalamus) are transcribed. Our findings also reveal that, besides the GABAergic phenotype, Otx $2^{-}$thalamic cell patches exhibit additional similarities in gene expression profile with the pretectum but not with the prethalamus or the basal telencephalon. Indeed, they show expression of Pax3, Pax7, and Lim1 but never that of $D l x 1$ and $D l \times 5$. Thus, although not conclusive, this observation suggests that lack of Otx2 in the thalamus is reflected in the generation of GABAergic neurons, which, on the basis of their gene expression profile, appear more similar to those of the pretectum than to those of the prethalamus or basal telencephalon.

However, although in the pretectum Otx2 is normally coexpressed with Pax3, Pax7, Lim1, and Mash1, our data suggest that, in the thalamus, Otx2 is required to repress these genes. A likely possibility reconciling this apparent contrast is that activating or repressing properties of Otx2 might depend on the territorial context in which it is expressed. In agreement with this, Otx2 plays different roles in different tissues during embryonic development (Acampora and Simeone, 1999; Acampora et al., 2001; Simeone et al., 2002). In particular, Otx2 is required to control identity and fate of ventral progenitors generating dopaminergic neurons in the midbrain (Puelles et al., 2003, 2004; Vernay et al., 2005; Prakash et al., 2006). In this context, we have shown that Grg4 (groucho-related gene), also expressed in the ventral midbrain, may interact with both Otx1 and Otx2 proteins and convert them from activators to repressors (Puelles et al., 2004). Thus, it is conceivable that Otx2 may convert its transcriptional properties (from activator to repressor and vice versa) by interacting with specific co-repressing or coactivating partner(s) expressed in restricted brain areas.

Here, we studied the role of Otx 2 in the thalamus and have shown that its ablation generates the activation of Pax3, Pax7, Lim1, Mash1, and GABAergic fate switch, whereas none of the genes whose expression is affected by the lack of Otx 2 in the ventral midbrain (Puelles et al., 2003, 2004) is activated in the Otx2 ${ }^{-}$ patches. In this context, our preliminary results aimed at detecting the role of Otx2 in the pretectal area, which generates GABAergic neurons and in which Otx2 is coexpressed with Pax3, Pax7, Lim1, and Mash1 (supplemental Fig. S1, available at www.jneurosci.org as supplemental material), show that severe reduction of Otx 2 in this region results in an increase of GABAergic neurons, thus suggesting that Otx2 in the pretectum might represent a mild negative modulator of the GABAergic fate. Thus, our data and those reported previously indicate that, depending on the brain territory, Otx2 is required to control identity and fate of different regionally restricted pools of progenitors. In this context, a crucial issue to be addressed will be to dissect in vivo the molecular basis of this regionally restricted differential control exerted by Otx2.

Finally, because the Otx ${ }^{+}$thalamic territory surrounding the Otx $2^{-}$patches develops normally and Shh expression at the zli is unaffected, it is likely that the abnormalities reported in this study reflect the lack of a cell-autonomous control by Otx2.

\section{Otx 2 controls proliferating activity of thalamic progenitors}

An additional consequence of Otx 2 inactivation is the generation of cell masses distributed within the mantle zone of the thalamus at late gestation. Analysis of proliferating activity indicates that the number of Otx $2^{-}$progenitors in $\mathrm{S}\left(\mathrm{BrdU}^{+}\right)$and $\mathrm{M}(\mathrm{Ph}-$ $\mathrm{H}^{+}{ }^{+}$) phases is increased by approximately twofold when compared with the Otx $2^{+}$progenitors of control embryos. This increase in proliferating activity is particularly evident between E12 and E14 but, at later stages, is less evident but reproducibly higher than in control embryos. In the patches, the ratio between Otx $2^{-}$ dividing progenitors $\left(\mathrm{Ph}-\mathrm{H}_{3}^{+}\right)$and those in $\mathrm{S}$ phase $\left(\mathrm{BrdU}^{+}\right)$is essentially unaltered, leading us to assume that the progression from $S$ to $M$ phase is unaffected and that size expansion of the patches would be attributable to altered postmitotic transition of Otx $2^{-}$progenitors. In this case, a higher proportion of Otx $2^{-}$ progenitors should reenter the cell cycle and be responsible for local and vigorous overgrowth. Whether this impairment may be considered as a somatic pretumorigenic event remains to be clarified, but certainly the outcome of this strong hyperplasia may be dramatic. However, to our current knowledge, no proliferationsuppressing activity has been documented for Otx2 in humans, although a similar effect on proliferation has already been described in retinal progenitors of $O t \times 1^{-1-} ; O t \times 2^{+/-}$doublemutant embryos (Martinez-Morales et al., 2001).

However, it has been reported recently that Otx2 is a medulloblastoma oncogene (Boon et al., 2005; Di et al., 2005). In this tumor, Otx2 is remarkably overexpressed, but, in the thalamus of this conditional mutant, the lack of Otx2 correlates with increased proliferation. This suggests that, depending on the expression level, Otx2 may have different roles in proliferation: its overexpression may result in a tumorigenic event, although its absence may be correlated to hyperplasia. Moreover, these findings suggest that altered proliferation may also be subject to the territory in which Otx2 expression is either lost or enhanced.

Future experiments designed to study the effect of both overexpression and ablation of Otx2 in specific cell types and at different stages should provide new insights on its role in cell proliferation in normal and pathological contexts.

\section{References}

Acampora D, Simeone A (1999) Understanding the roles of Otx1 and Otx2 in the control of brain morphogenesis. Trends Neurosci 22:116-122.

Acampora D, Gulisano M, Broccoli V, Simeone A (2001) Otx genes in brain morphogenesis. Prog Neurobiol 64:69-95.

Agarwala S, Sanders TA, Ragsdale CW (2001) Sonic hedgehog control of size and shape in midbrain pattern formation. Science 291:2147-2150.

Bennett MR, Balcar VJ (1999) Forty years of amino acid transmission in the brain. Neurochem Int 35:269-280.

Boon K, Eberhart CG, Riggins GJ (2005) Genomic amplification of Orthodenticle Homologue 2 in medulloblastomas. Cancer Res 65:703-707.

Briscoe J, Ericson J (2001) Specification of neuronal fates in the ventral neural tube. Curr Opin Neurobiol 11:43-49.

Briscoe J, Pierani A, Jessell TM, Ericson J (2000) A homeodomain protein code specifies progenitor cell identity and neuronal fate in the ventral neural tube. Cell 101:435-445. 
Casarosa S, Fode C, Guillemot F (1999) Mash1 regulates neurogenesis in the ventral telencephalon. Development 126:525-534.

Cheng L, Arata A, Mizuguchi R, Qian Y, Karunaratne A, Gray PA, Arata S, Shirasawa S, Bouchard M, Luo P, Chen CL, Busslinger M, Goulding M, Onimaru H, Ma Q (2004) Tlx3 and Tlx1 are post-mitotic selector genes determining glutamatergic over GABAergic cell fates. Nat Neurosci 7:510-517.

Di C, Liao S, Adamson DC, Parrett TJ, Broderick DK, Shi Q, Lengauer C, Cummins JM, Velculescu VE, Fults DW, McLendon RE, Bigner DD, Yan $\mathrm{H}$ (2005) Identification of OTX2 as a medulloblastoma oncogene whose product can be targeted by all-trans retinoic acid. Cancer Res 65:919-924.

Edlund T, Jessell TM (1999) Progression from extrinsic to intrinsic signalling in cell fate specification: a view from the nervous system. Cell 96:211-224.

Figdor MC, Stern CD (1993) Segmental organization of embryonic diencephalon. Nature 363:630-634.

Fode C, Ma Q, Casarosa S, Ang SL, Anderson DJ, Guillemot F (2000) A role for neural determination genes in specifying the dorsoventral identity of telencephalic neurons. Genes Dev 14:67-80.

Fremeau Jr RT, Voglmaier S, Seal RP, Edwards RH (2004) VGLUTs define subsets of excitatory neurons and suggest novel roles for glutamate. Trends Neurosci 27:98-103.

Hynes M, Rosenthal A (1999) Specification of dopaminergic and serotonergic neurons in the vertebrate CNS. Curr Opin Neurobiol 9:26-36.

Jessell TM (2000) Neuronal specification in the spinal cord: inductive signals and transcriptional codes. Nat Rev Genet 1:20-29.

Kiecker C, Lumsden A (2004) Hedgehog signaling from the ZLI regulates diencephalic regional identity. Nat Neurosci 7:1242-1249.

Lumsden A (1990) The cellular basis of segmentation in the developing hindbrain. Trends Neurosci 13:329-335.

Lumsden A, Krumlauf R (1996) Patterning the vertebrate neuraxis. Science 274:1109-1115.

Luskin MB, Parnavelas JG, Barfield JA (1993) Neurons, astrocytes, and oligodendrocytes of the rat cerebral cortex originate from separate progenitor cells: an ultrastructural analysis of clonally related cells. J Neurosci 13:1730-1750.

Marin O, Rubenstein JL (2002) Patterning, regionalization, and cell differentiation in the forebrain. In: Mouse development, Vol 5, pp 75-106. San Diego: Academic.

Martinez-Morales JR, Signore M, Acampora D, Simeone A, Bovolenta P (2001) Otx genes are required for tissue specification in the developing eye. Development 128:2019-2030.

Mione MC, Danevic C, Boardman P, Harris B, Parnavelas JG (1994) Lineage analysis reveals neurotransmitter (GABA or glutamate) but not calcium-binding protein homogeneity in clonally related cortical neurons. J Neurosci 14:107-123.

Miyoshi G, Bessho Y, Yamada S, Kageyama R (2004) Identification of a novel basic helix-loop-helix gene, Heslike, and its role in GABAergic neurogenesis. J Neurosci 24:3672-3682.

Muzio L, DiBenedetto B, Stoykova A, Boncinelli E, Gruss P, Mallamaci (2002) A Conversion of cerebral cortex into basal ganglia in $\operatorname{Emx2}(-/-)$ Pax6(Sey/Sey) double-mutant mice. Nat Neurosci 5:737-745.

Parras CM, Schuurmans C, Scardigli R, Kim J, Anderson DJ, Guillemot F (2002) Divergent functions of the proneural genes Mash1 and Ngn2 in the specification of neuronal subtype identity. Genes Dev 16:324-338.

Prakash N, Brodski C, Naserke T, Puelles E, Gogoi R, Hall A, Panhuysen M,
Echevarria D, Sussel L, Weisenhorn DM, Martinez S, Arenas E, Simeone A, Wurst W (2006) A Wnt1-regulated genetic network controls the identity and fate of midbrain-dopaminergic progenitors in vivo. Development 133:89-98.

Puelles L, Rubenstein JL (2003) Forebrain gene expression domains and the evolving prosomeric model. Trends Neurosci 26:469-476.

Puelles E, Acampora D, Lacroix E, Signore M, Annino A, Tuorto F, Filosa S, Corte G, Wurst W, Ang SL, Simeone A (2003) Otx dose-dependent integrated control of antero-posterior and dorso-ventral patterning of midbrain. Nat Neurosci 6:453-460.

Puelles E, Annino A, Tuorto F, Usiello A, Acampora D, Czerny T, Brodski C, Ang SL, Wurst W, Simeone A (2004) Otx2 regulates the extent, identity and fate of neuronal progenitor domains in the ventral midbrain. Development 131:2037-2048.

Rubenstein JL, Shimamura K, Martinez S, Puelles L (1998) Regionalization of the prosencephalic neural plate. Annu Rev Neurosci 21:445-477.

Schuurmans C, Armant O, Nieto M, Stenman JM, Britz O, Klenin N, Brown C, Langevin LM, Seibt J, Tang H, Cunningham JM, Dyck R, Walsh C, Campbell K, Polleux F, Guillemot F (2004) Sequential phases of cortical specification involve Neurogenin-dependent and -independent pathways. EMBO J 23:2892-2902.

Shi SR, Key ME, Kalra KL (1991) Antigen retrieval in formalin-fixed, paraffin-embedded tissues: an enhancement method for immunohistochemical staining based on microwave oven heating of tissue sections. J Histochem Cytochem 39:741-748.

Shi SR, Cote RJ, Taylor CR (1997) Antigen retrieval immunohistochemistry: past, present, and future. J Histochem Cytochem 4:327-343.

Shi SR, Cote RJ, Taylor CR (2001) Antigen retrieval techniques: current perspectives. J Histochem Cytochem 49:931-938.

Simeone A (1999) Detection of mRNA in tissue sections with radiolabelled riboprobes. In: In situ hybridization. A practical approach (Wilkinson DG, ed), Ed 2, pp 69-86. Oxford: Oxford UP.

Simeone A (2005) Genetic control of dopaminergic neuron differentiation. Trends Neurosci 28:62-65.

Simeone A, Acampora D, Gulisano M, Stornaiuolo A, Boncinelli E (1992) Nested expression domains of four homeobox genes in developing rostral brain. Nature 358:687-690.

Simeone A, Acampora D, Mallamaci A, Stornaiuolo A, D'Apice MR, Nigro V, Boncinelli E (1993) A vertebrate gene related to orthodenticle contains a homeodomain of the bicoid class and demarcates anterior neuroectoderm in the gastrulating mouse embryo. EMBO J 12:2735-2747.

Simeone A, Puelles E, Acampora D (2002) The Otx family. Curr Opin Genet Dev 12:409-415.

Toresson H, Potter SS, Campbell K (2000) Genetic control of dorsal-ventral identity in the telencephalon: opposing roles for Pax6 and Gsh2. Development 127:4361-4371.

Vernay B, Koch M, Vaccarino F, Briscoe J, Simeone A, Kageyama R, Ang SL (2005) Otx2 regulates subtype specification and neurogenesis in the midbrain. J Neurosci 25:4856-4867.

Wolpert L (1969) Positional information and the spatial pattern of cellular differentiation. J Theor Biol 25:1-47.

Wurst W, Bally-Cuif L (2001) Neural plate patterning: upstream and downstream of the isthmic organizer. Nat Rev Neurosci 2:99-108.

Ye W, Shimamura K, Rubenstein JL, Hynes MA, Rosenthal A (1998) FGF and Shh signals control dopaminergic and serotonergic cell fate in the anterior neural plate. Cell 93:755-766. 